\title{
Effects of Collar Permeability on the Field Quality of the Large Aperture Quadrupoles for the LHC
}

\author{
N. Catalan Lasheras, B. Auchmann, G. Kirby, K.-H. Mess, R. Ostojic, and S. Russenschuck
}

\begin{abstract}
The LHC contains a number of large aperture quadrupoles (MQY) in the insertions. The acceptance of these magnets was based on warm magnetic measurements performed before delivery to CERN. During the series production of the MQY quadrupoles, the permeability of the collars drifted from the nominal value, and effects on the transfer function and multipole components became evident. To study the effects on the magnetic field, variable permeability of the stainless-steel collars as a function of local field and temperature was introduced into a numerical model. Comparing the results with measured data, we could isolate the contribution of permeability deviation on the magnetic field quality. The extrapolation of transfer function and field multipoles to operating temperature and current gives the necessary offsets, which are compared with measurements on a reduced set of magnets.
\end{abstract}

Index Terms-LHC, magnetic field measurements, permeability.

\section{INTRODUCTION}

$\mathbf{T}$ HE special superconducting quadrupoles for the LHC insertions (MQY) feature a large aperture $(70 \mathrm{~mm})$ and a nominal gradient of $160 \mathrm{~T} / \mathrm{m}$ at $4.5 \mathrm{~K}$ [1]. Thirty of these magnets were built by ACCEL (Germany). As part of the quality assurance, the MQY magnets were measured at room temperature using a rotating coil measurement system at $17 \mathrm{~mm}$ described in [2]. These measurements served to ensure the uniformity of the production and to predict the field quality of each magnet at nominal operating conditions.

Half way through the production, we observed abnormal field multipoles in some magnets that could not be explained by production tolerances. This problem was already seen in the LHC arc quadrupoles and was traced back to abnormally high permeability of the collars [3], [4]. To verify this, special measurements were performed on a MQY magnet at $4.5 \mathrm{~K}$. The values of the transfer function measured at room temperature and at $4.5 \mathrm{~K}$ did not follow the correlation established for the earlier production magnets. Indeed, it was observed that the perturbations of the field gradient decreased above $100 \mathrm{~A}$, which was consistent with the fact that permeability of stainless-steel collars decreases with field.

Once it was shown that the quality of the magnetic field was restored at operating conditions, we proceeded with the production of the MQY magnets using all available collars even though their permeability was outside specifications.

As the overall warm-to-cold correlation was not meaningful anymore, most of the magnets with abnormal transfer function

Manuscript received August 23, 2007.

The authors are with CERN, Accelerator Technology Department, Geneva 23, 1211 Switzerland (e-mail: Nuria.Catalan.Lasheras@cern.ch).

Color versions of one or more of the figures in this paper are available online at http://ieeexplore.ieee.org.

Digital Object Identifier 10.1109/TASC.2008.922245 were systematically measured in operating conditions. However, for practical reasons a small number of magnets could not be measured. In this paper we present an approach for estimating the magnetic field of MQY magnets in operating conditions based on collar permeability and warm measurements.

\section{EfFects of the PeRmeAbility on the Magnetic FiELD}

The permeability $\mu$ of a homogeneous, isotropic material is defined as

$$
B=\mu H=\mu_{0}(1+\chi) H
$$

where $B$ is the magnetic flux density, $\mu_{0} H$ the magnetic field strength, and $\chi$ the magnetic susceptibility. The relative permeability is $\mu_{r}=\mu / \mu_{0}=1+\chi$. The collars used for assembly of the LHC magnets are made of austenitic steel with a relative permeability of $1.003 \pm 0.002$. The design of the magnets assumes a very small contribution to the field from the collars. A change in collar permeability will induce slightly different field strength and thus different field harmonic components. Assuming the quadrupole symmetry is still preserved, the effects of changes in permeability will be most visible in the «allowed» harmonics of low-order like $b_{2}$ or $b_{6}$. These harmonics are otherwise robust against changes in geometry, the effects of which, are well known [6]. Changes of non-allowed harmonics are also possible if collars of different permeability are not distributed homogeneously around the coils. We should note that the magnetic field of the LHC magnets needs to be very homogeneous and the field absolute harmonics are required to be less than $10^{-4}$ of the main field (one «unit») at $17 \mathrm{~mm}$ reference radius.

\section{A. Field Dependent Permeability}

In practice, the relation (1) is not linear and permeability is not constant but varies with magnetic field and, to a lesser extent, with temperature and stress. Measurements of stainless steel permeability exist for fields as low as $0.1 \mathrm{~T}$, [7]. Fig. 1 shows measurements done as part of the steel qualification for the collars of LHC superconducting magnets, with data for 23 different collars measured in fields from 0.1 to $8 \mathrm{~T}$ at $4 \mathrm{~K}$. Relative permeability decreases with magnetic field but reaches values as large as 1.04 at low current. Note that the spread of measured values is higher at low fields. At $8 \mathrm{~T}$, all collars have practically the same permeability, within the tolerances specified for LHC magnets $\left(\mu_{r}<1.003 \pm 0.002\right)$.

We need to clarify the use of permeability at three different points during the production of MQY.

1) The ROXIE [5] simulations done during the design phase of the magnet and used to establish the tolerances in the field harmonics assume a constant relative permeability of 1.003 . 


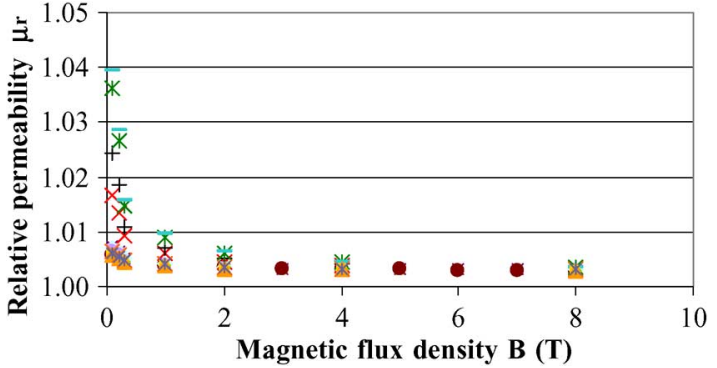

Fig. 1. Measurements of collar relative permeability vs. magnetic flux density.

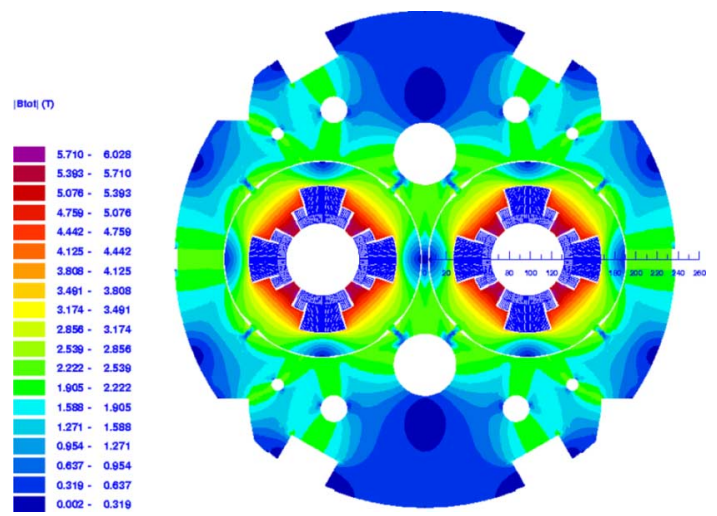

Fig. 2. Magnetic density flux on the MQY yoke and collars at 3 A.

2) When measuring permeability, most permeameters apply an external field of $0.1 \mathrm{~T}$. This is indeed the case at ACCEL who provided the measurements of the collars used in the fabrication of MQY magnets.

3) The field in the collars when powered during room temperature magnetic measurements $(3 \mathrm{~A})$, is very small and ranges from 1 to $50 \cdot 10^{-4} \mathrm{~T}$ (Fig. 2). The permeability varies considerably in the collar and cannot be considered constant.

To evaluate the change in harmonics, we consider in ROXIE the permeability as a function of the field.

\section{B. ROXIE Model}

By analyzing the measured data in [7], we assumed a simple power law for the susceptibility:

$$
\begin{aligned}
& \chi=\chi_{0} \cdot B^{-\alpha\left(\chi_{0}\right)} \\
& \alpha=-7765.1 \chi_{0}^{2}+175.8 \chi_{0}-0.398
\end{aligned}
$$

where $\chi_{0}$ is the susceptibility at $1 \mathrm{~T}$, and $\alpha$ a parabolic function of $\chi_{0}$. When comparing the fitted model values to the measurements, the largest difference is $15 \%$.

We introduced in ROXIE the dependence of permeability with field extrapolated to $5 \cdot 10^{-3} \mathrm{~T}$. We performed simulations with a current of $3 \mathrm{~A}$, conditions at which the magnetic measurements for acceptance are done. We then ran simulations at the nominal operating currents for MQY magnets in the LHC at injection (176 A) and flat-top (3600 A). The results are shown in Fig. 3.

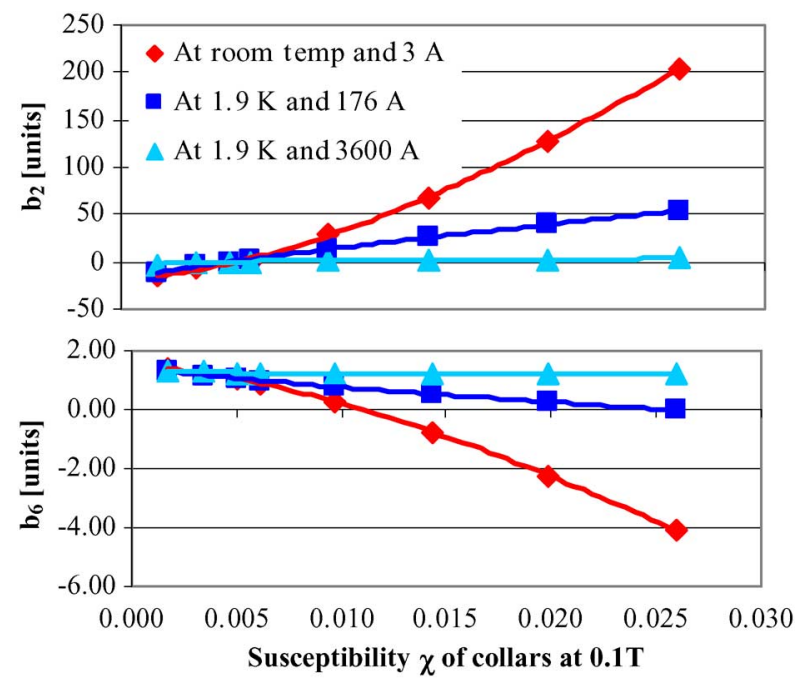

Fig. 3. Variation of $b_{2}$, and $b_{6}$ versus susceptibility $\chi$ measured at $0.1 \mathrm{~T}$.

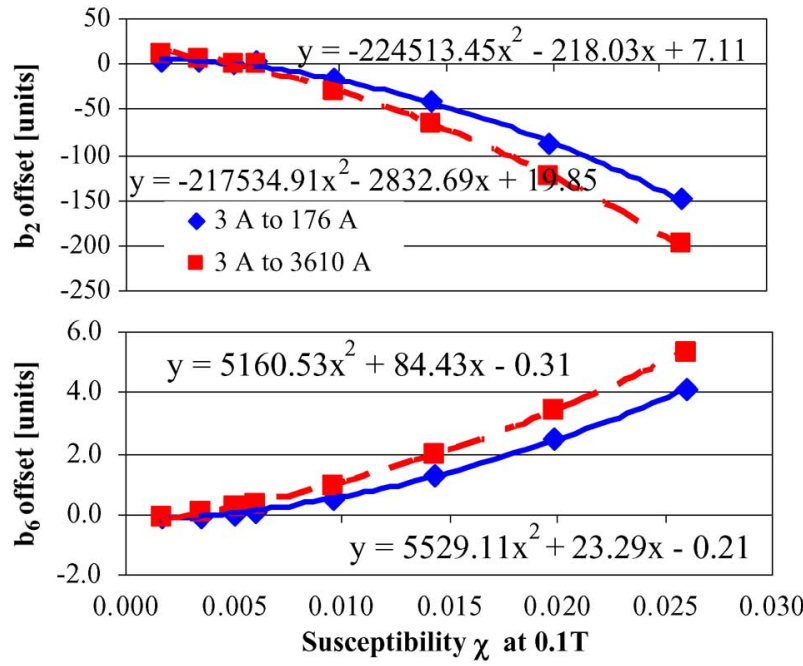

Fig. 4. Offsets for warm-cold correlation for $b_{2}$ and $b_{6}$ at injection (176 A) and top energy (3610 A) for the MQY magnet as given by ROXIE simulations. Offsets are given as a function of $\chi_{0}$.

We observed the dependence of harmonics with susceptibility for each case. Note that the nominal values of the transfer function were taken for $\mu_{r}=1.003$ which corresponds to the specifications of the collar stainless steel.

The variations of transfer function and sextupole components at $3 \mathrm{~A}$ are significant but decrease with current. At flat top the harmonics are almost constant with collar permeability. A magnet assembled with collars with $\mu_{\mathrm{r}}=1.01$ deviates by $39 \cdot 10^{-4}$ from the nominal field at 3 A but only $2.3 \cdot 10^{-4}$ at 3610 A. For comparison, the accuracy of the transfer function measurement is evaluated to about $10 \cdot 10^{-4}$.

From the simulations we estimated the correction values that need to be added to the $b_{2}$ and $b_{6}$ harmonics measured at warm to obtain their value at $176 \mathrm{~A}$ and $3610 \mathrm{~A}$. Unlike geometric errors, we do not find a constant offset but the correction depends also on the reference susceptibility as shown in Fig. 4. This shift accounts only for the change of the susceptibility with current. Other offsets are necessary to account for changes in geometry during contraction or due to persistent currents. 


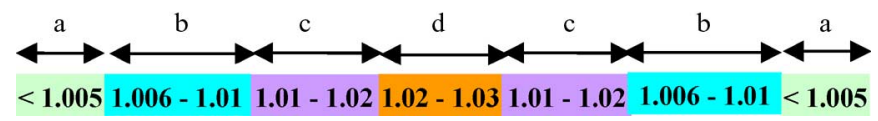

Fig. 5. Distribution of collars along MQY collared aperture. Collars are classified according to a range of permeability. Collars inside tolerance are assembled near ends. The values communicated by ACCEL are a, b, c and d.

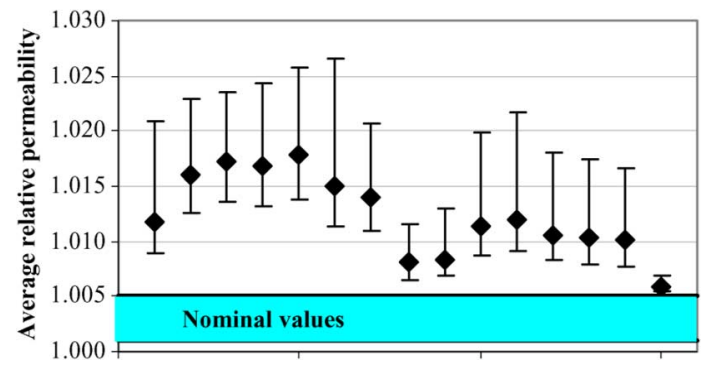

Fig. 6. Permeability of 15 assemblies deduced from the collar distribution communicated by ACCEL.

\section{EXPERIMENTAL DATA FROM MQY PRODUCTION}

\section{A. Permeability Measurements}

From the last section, it is clear that we need to know the permeability, measured at $0.1 \mathrm{~T}$ in ACCEL to retrieve the offset and estimate the transfer function and harmonics at $176 \mathrm{~A}$ and 3610 A from the warm magnetic measurements. However, permeability measurements of the collars were not systematically done during magnet production. Only after the first indications that permeability may be an issue (which occurred after completion of a third of the production), was permeability of the collars measured systematically at reception. In subsequent magnets, the collars were grouped according to a given range, and distributed along the collared coil with the highest permeability in the central part (Fig. 5).

The estimate of the mean permeability of the aperture was very approximate with large error bars (up to 0.012). In addition to that, only 15 out of 60 assembled apertures $(25 \%)$ were measured. Fig. 6 shows the mean relative permeability along the magnet for these apertures. Error bars represent the maximum and minimum value of the permeability when taking the higher and lower limits of the ranges used at ACCEL.

\section{B. Estimate of the Permeability}

The mean permeability in a magnet can be estimated on the basis of the deviation of $b_{2}$ and $b_{6}$ from the nominal value measured at room temperature. Reference [4], uses a linear approximation; we use a parabolic relation, previously obtained by the ROXIE model. From the $b_{n}(\chi)$ relations in Fig. 3 we solve for the measured $b_{n}$ and obtain two independent estimates of the susceptibility $\chi_{b 2}$ and $\chi_{b 6}$. As the reference for the transfer function, we used the average value of four apertures which were assembled using collars with nominal permeability. To weight the estimates, we used the cross-correlation between the estimated susceptibility $\chi_{b n}$ and the values measured at ACCEL $\chi$.

$$
\chi=\frac{\rho\left(\chi, \chi_{b 2}\right) \chi_{b 2}+\rho\left(\chi, \chi_{b 6}\right) \chi_{b 6}}{\rho\left(\chi, \chi_{b 2}\right)+\rho\left(\chi, \chi_{b 6}\right)} .
$$

with $\rho\left(\chi, \chi_{b 2}\right)=0.873$ and $\rho\left(\chi, \chi_{b 6}\right)=0.950$. In Fig. 7 , we compare the estimated permeability with the values measured

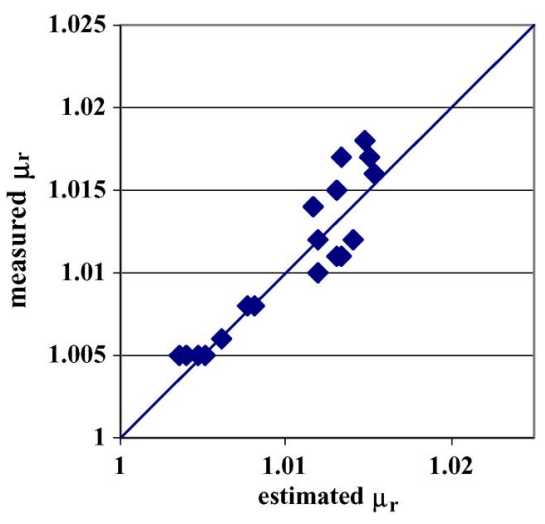

Fig. 7. Comparison of the measured and estimated permeability for $15 \mathrm{MQY}$ collared coils.
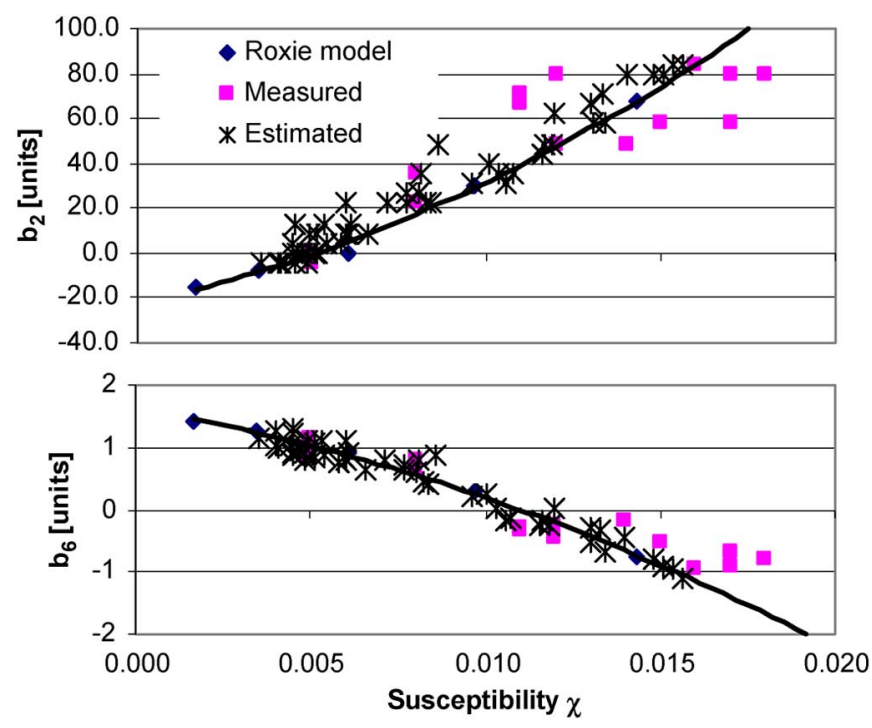

Fig. 8. Final value of $\chi$ for the whole production of MQY compared with the models for $b_{2}$ and $b_{6}$. Magnets for which susceptibility was measured in ACCEL are shown as squares.

in ACCEL, which coincide within 0.0035. Indeed, this error is smaller than the expected error due to sorting of the collars.

The final $\chi$ calculated from (3) for each MQY aperture is shown in Fig. 8 and compared with the ROXIE model which takes into account only $b_{2}$ or $b_{6}$. Superimposed are the magnets for which the susceptibility was measured in ACCEL. As already indicated by the correlation, the agreement is better for $\chi$ based on the $b_{6}$ harmonic. In both cases, the model agrees very well with the measured values so that we can be reasonably confident on the susceptibility value we use.

\section{Warm to Cold Correlation}

Twelve magnets were fully measured in operating conditions $(4.5 \mathrm{~K})$. Before applying the offsets to the magnets that were only measured at room temperature, we could verify the accuracy of our models by comparing the predictions with these measurements. This is done in Fig. 9. The model fits the measurements quite well, although the measurements have a larger spread. The additional offset of $b_{6}$ comes from the persistent currents which account for about 4 units [8]. The error bar for the susceptibility $\chi$ is about 0.0035 in this figure. The accuracy 

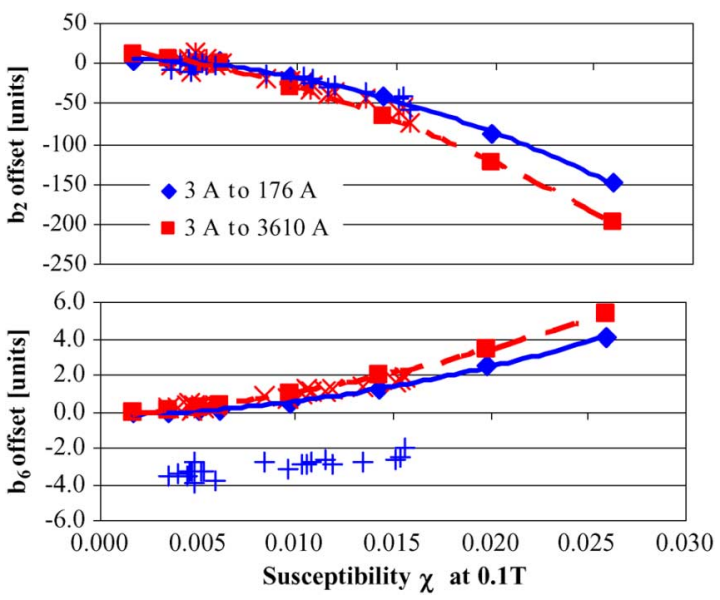

Fig. 9. Offsets between warm and cold harmonics as calculated by ROXIE (line) and as measured (crosses).

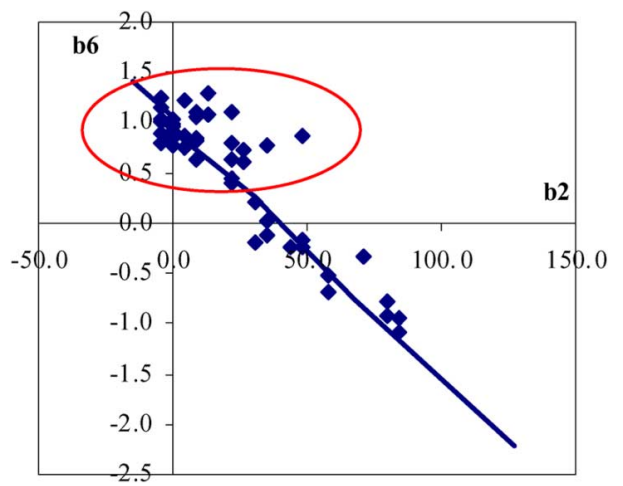

Fig. 10. $b_{2}-b_{6}$ correlation for the MQY production. The line is the $b_{2}-b_{6}$ relationship given by ROXIE.

of the warm and cold measurements contributes to an error bar of about 7 units for $b_{2}$, and 0.14 units for $b_{6}$.

\section{Correction on MQY Series Magnetic Quality}

All our calculations assumed that most of the deviation from nominal values is due to the magnetic permeability. However, other effects contribute to the errors, especially for $b_{2}$. Fig. 10 shows the $b_{2}$ and $b_{6}$ harmonics measured during the MQY production. We observe a cloud of uncorrelated values and a tail stretching to negative values of $b_{6}$. The uncorrelated cloud includes the natural spread of $b_{2}$ and $b_{6}$ due to manufacturing tolerances and measurement errors. The effect of permeability inside this area is masked. For collared coils with a low $b_{6}$, a clear correlation between $b_{2}$ and $b_{6}$ indicates a general increase of field that can be attributed to a change in permeability. According to the ROXIE model, the minimum permeability that will result in a clearly modified harmonic $\left(b_{6}<0.5\right.$ units $)$ is $\mu_{r}=1.0086$.

We assume that the points inside the uncorrelated cloud in Fig. 10 represent the magnets without permeability deviation and we use them as a healthy sample to calculate the average and natural spread of the $b_{2}$ and $b_{6}$ harmonics during production due to geometric tolerances (Fig. 11)

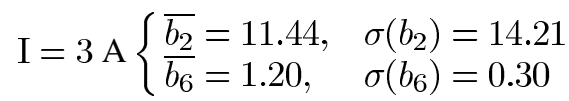

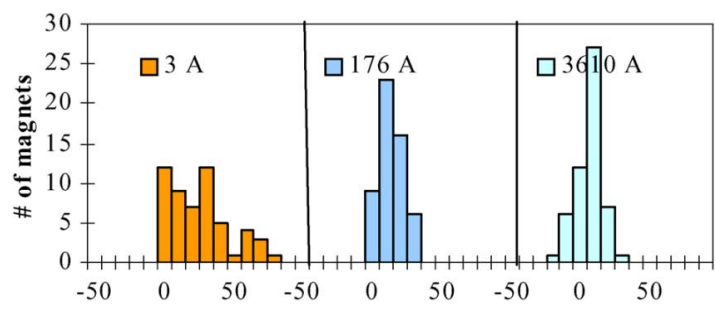

Fig. 11. Distribution of $b_{2}$ of the MQY series at 3, 176 and 3610 A.

The positive average for $b_{2}$ indicates that permeability still influences our sample. Indeed, after correction for permeability, the spread of $b_{2}$ at $176 \mathrm{~A}$ becomes smaller. This spread decreases further for $3610 \mathrm{~A}$ :

$$
\begin{gathered}
\mathrm{I}=176 \mathrm{~A} \begin{cases}\bar{b}_{2}=8.62, & \sigma\left(b_{2}\right)=8.65 \\
\bar{b}_{6}=1.35, & \sigma\left(b_{6}\right)=0.18\end{cases} \\
\mathrm{I}=3610 \text { A } \begin{cases}\bar{b}_{2}=1.34, & \sigma\left(b_{2}\right)=9.24 \\
\bar{b}_{6}=1.70, & \sigma\left(b_{6}\right)=0.26\end{cases}
\end{gathered}
$$

These last values should be used as the natural average and standard deviation of the $b_{2}$ and $b_{6}$ harmonics for MQY magnets when setting the LHC in operating conditions.

\section{CONCLUSIONS}

The permeability of the collars affects significantly the magnetic filed quality of the MQY magnets when measured at room temperature and low current. After verifying that the field quality is restored in nominal operating conditions $(4.5 \mathrm{~K}$, $160 \mathrm{~T} / \mathrm{m}$ ), we decided to continue magnet production using collars having higher than specified permeability. We worked out a model of the dependence of permeability with field, which was used in ROXIE calculations to reproduce the experimental results. The model confirmed that the large differences observed between magnets at low field disappear at high field.

Using this model we estimated the average value of the collar permeability of each magnet on the basis of the $b_{2}$ and $\mathrm{b}_{6}$ harmonics measured at room temperature, with a precision better than given by standard permeability meters. These values were then used to extrapolate the room temperature measurements to operating conditions. The spread of transfer function as well as $b_{6}$ for the full series of MQY magnets became after correction much smaller and comparable to other LHC magnets. This should ease early commissioning with beam and LHC operation.

\section{REFERENCES}

[1] O. S. Brüning, (ed.) et al., "LHC Design Report vol. I. The LHC Main Ring," CERN-2004-003 [Online]. Available: http://www.ab-div.web. cern.ch/ab-div/Publications/LHC-DesignReport.html

[2] J. García-Pérez et al., "Performance of the Room Temperature Systems for Magnetic Field Measurements of the LHC Superconducting Magnets," CERN, CERN-LHC-Project-Report-873, 2006.

[3] F. Simon, Private Communication..

[4] P. Hagen and E. Todesco, "Impact of Magnetic Permeability of the Collars and Warm-Cold Correlations on Magnetic Field in the LHC Main Quadrupoles," CERN, LHC-Project-Report-868, 2006.

[5] S. Russenschuck, "ROXIE: A Computer Code for the Integrated Design of Accelerator Magnets," CERN, LHC-Project-Report-276, 1999.

[6] J. Lucas, Electromagnetic Design of the MQM Quadrupole CERN, CERN-OPEN-2000-157, 1999.

[7] G. Peiro, "LHC-MQ Collars Permeability Measurements," EDMS 496251.

[8] S. Sanfilippo, "Magnetic performance of the main superconducting magnets for the LH,", submitted for publication. 